\title{
El análisis financiero como herramienta para la toma de decisiones
}

Main Financial analysis as a tool for decision making

\author{
Vanessa K. Hilario García ${ }^{a}$, Omar Castro Pérez ${ }^{b}, J$. Carlos Mendoza Hernández ${ }^{b}$, Irma Isabel \\ de León Vázquez ${ }^{c}$, Daniel Vélez Díaz ${ }^{d}$, Delia Iliana Tapia Castillo ${ }^{e}$
}

\begin{abstract}
:
A study was carried out on a transport company, the main objective is financial analysis and how it influences decision making. Using a mixed methodology with a descriptive approach comparing two periods 2017 and 2018 respectively. Based on the population of the business unit. The financial ratios applied in the two-year profit and loss statements were used as instruments. The results were measured thanks to the application of the basic financial reasons. It is concluded that the use of financial tools serves to know a better panorama of the organization.
\end{abstract}

Keywords:

Financial Analysis, decisión making, financial ratios, profit and los

\section{Resumen:}

Se realizó un estudio a una empresa de transporte, el principal objetivo es el análisis financiero y cómo influye en la toma de decisiones. Empleando una metodología mixta con un enfoque descriptivo comparando dos periodos 2017 y 2018 respectivamente. Tomando como base la población de la unidad de negocio. Se utilizaron como instrumentos las razones financieras aplicadas en el estado de pérdidas y ganancias de los dos años. Los resultados se lograron medir gracias a la aplicación de las razones financieras básicas. Se concluye que la utilización de herramientas financieras sirva para conocer un mejor panorama de la organización.

\section{Palabras Clave:}

Análisis financiero, toma de decisiones, razones financieras, estado de pérdidas y ganancias

\section{1.- Introducción}

El presente reporte técnico es una investigación que tiene por objetivo evaluar los resultados del análisis financiero de la Empresa de Transporte, mediante herramientas financieras y su influencia en la toma de decisiones durante el periodo $2017-2018$.

Es importante conocer cómo se encuentra la situación económica de una empresa para saber cuáles son sus debilidades, en base a sus datos históricos para pronosticar posibles escenarios futuros, tomando medidas correctivas a tiempo, aplicarlas de manera eficiente, así como identificar oportunidades de mejora dentro de la organización, logrando de esta manera unas finanzas sanas.

\section{2.- Planteamiento del Problema.}

Actualmente es de suma importancia tener una correcta administración financiera dentro de la empresa sin importar su tamaño, giro o actividad económica que estas desarrollen.

\footnotetext{
${ }^{a}$ Autor de Correspondencia, Universidad Autónoma del Estado de Hidalgo, Escuela Superior de Tlahuelilpan, Alumno de la Licenciatura en Administración, Email: hi338073@uaeh.edu.mx

${ }^{\mathrm{b}}$ Universidad Autónoma del Estado de Hidalgo, Escuela Superior de Tlahuelilpan, Alumno de la Licenciatura en Administración, Email: ca339756@uaeh.edu.mx,me338914@uaeh.edu.mx

c Universidad Autónoma del Estado de Hidalgo, Escuela Superior de Tlahuelilpan, Profesor por Asignatura de la Licenciatura en Administración, Email: irmalv@uaeh.edu.mx

${ }^{d}$ Universidad Autónoma del Estado de Hidalgo, Escuela Superior de Tlahuelilpan, Profesor Investigador, Email: daniel@uaeh.edu.mx

e Universidad Autónoma del Estado de Hidalgo, Escuela Superior de Tlahuelilpan, Profesor Investigador de la Licenciatura en Administración, Email: itapia@uaeh.edu.mx
} 
La alta gerencia muchas veces desconoce el verdadero significado de las finanzas, y no hace uso de las herramientas financieras en las que se pueden apoyar, y de esta manera se toman decisiones solo por instinto y sin fundamento.

La contabilidad representa la realidad económica y financiera de la empresa. Las empresas están expuestas a retos como los altos costos de producción, falta de innovación tecnológica y en especial, la problemática financiera. Igualmente, el desconocimiento de herramientas financieras provoca que no lleven un análisis de su información financiera y económica contenida en los estados financieros.

\section{3.- Pregunta de investigación.}

¿A través de qué herramienta una empresa de transporte puede obtener mejores resultados en el análisis financiero que influya en la toma de decisiones?

\section{4.- Justificación.}

La investigación es de suma importancia, en toda organización es indispensable llevar una correcta administración financiera, un análisis adecuado de las finanzas proporcionará información que será de gran ayuda para poder hacer un diagnóstico eficiente sobre cuál es la situación económica actual de la organización.

El análisis permitirá conocer más a detalle cual es la salud financiera de la organización, siendo posible detectar deficiencias y patrones negativos, y poder aplicar oportunamente medidas correctivas a problemas potenciales que pueden afectar el funcionamiento de la empresa. De esta manera el administrador financiero podrá aportar estrategias para lograr un manejo correcto de las finanzas y estimar predicciones en el futuro.

Hacer una evaluación de los efectos obtenidos en la Empresa Transportista durante los ciclos 2017-2018, influirá y será de gran beneficio en la toma de decisiones.

\section{5.- Objetivo general.}

Evaluar los efectos del análisis financiero de la Empresa Transportista, mediante instrumentos financieros y su dominio en la toma de decisiones durante el periodo 2017 -2018 .

\section{6.- Objetivo específicos.}

Emplear el método de análisis vertical en los estados financieros de la Empresa, mediante herramientas e indicadores y su influencia en la toma de decisiones, durante el periodo 2017 - 2018.

Emplear el método de análisis horizontal en los estados financieros de la Empresa, mediante herramientas e indicadores y su influencia en la toma de decisiones, durante el periodo $2017-2018$.

Explicar los resultados obtenidos de los métodos de análisis financiero de la Empresa, mediante el análisis e interpretación financiera y su influencia en la toma de decisiones, durante el periodo $2017-2018$.

Plantear lineamientos económicos y financieros al Empresa y su influencia en la toma de decisiones, durante el periodo 2017 -2018.

\section{7.- Marco referencial}

\section{1.- Estados financieros.}

Los estados financieros son de mucha beneficio para las empresas, los cuales se disponen al término del periodo contable (considerado del $1^{\circ}$ de enero al 31 de diciembre), los cuales ayudan a conocer como se encuentra la situación actual de la organización y cuáles han sido los resultados de acuerdo a las actividades que se desempeñaron en el año.

Son documentos que se muestran en cantidades y por ellos son cuantitativos; los cuales se realizan cada determinado período de acuerdo a los datos historicos de la empresa, expresando cómo se encuentran las organizaciones económica y financieramente a una fecha determinada. (Galvez, 2013)

\subsection{1.- Clasificación de los estados financieros.}

Haciendo narración a Guadalupe Ochoa Setzer 2012 define estos conceptos de la siguiente manera:

\begin{tabular}{|l|l|}
\hline Estadis financieros & concepto \\
\hline Balance general & $\begin{array}{l}\text { Muestran cuales son los } \\
\text { bienes que posee la } \\
\text { empresa y cuales sus } \\
\text { deudas, asi como el } \\
\text { patrimonio }\end{array}$ \\
\hline
\end{tabular}




\begin{tabular}{|l|l|}
\hline Estado de resultados & $\begin{array}{l}\text { Expresa como se han ido } \\
\text { obteniendo los ingresos y } \\
\text { cuales han sido los costos } \\
\text { y gastos de la empresa }\end{array}$ \\
$\begin{array}{l}\text { Estado de cambio en la } \\
\text { situación financiera }\end{array}$ & $\begin{array}{l}\text { Muestra como han sido } \\
\text { conseguidos los recursos } \\
\text { para el ejercicio de la } \\
\text { organización, pueden ser } \\
\text { las utilidades del ejercicio } \\
\text { anterior o una forma de } \\
\text { financiamiento }\end{array}$ \\
\hline $\begin{array}{l}\text { Estado de variación en } \\
\text { el capital contable }\end{array}$ & $\begin{array}{l}\text { Muestra como han sido la } \\
\text { variaciones de la cuenta } \\
\text { de capital en un } \\
\text { determinado período }\end{array}$ \\
\hline $\begin{array}{l}\text { Estado de costo de } \\
\text { producción y costo de } \\
\text { producción de lo } \\
\text { vendido }\end{array}$ & $\begin{array}{l}\text { Destella la inversión para } \\
\text { cada una de las máquinas } \\
\text { que estan presentes en la } \\
\text { inversión de la materia } \\
\text { prima convertiendola en } \\
\text { un producto, y } \\
\text { posteriormente su venta a } \\
\text { este precio de costo }\end{array}$ \\
\hline
\end{tabular}

7.2.- Análisis e interpretación de los estados financieros.

El análisis de estados financieros es una parte integral e importante del campo más amplio del análisis de negocios, mediante el cual se puede evaluar el comportamiento operativo de una empresa mediante un diagnostico situacional actual y prever futuras complicaciones.

Nombrando a algunos autores relevantes se presentan las siguientes definiciones:

"Es un proceso en el cual se desintegra el fenomeno objeto de estudio en partes reales y su principal proposito es descubrir el origen de sus causas". (Armenteros, 2018)

"Proceso en el cual se seleccionan, relacionan y se evalua la información financiera mediante diferentes tipos de herramientas, obteniendo un fundamneto que permita tomar decisiones acertadad en el mundo de las finanzas". (Ochoa Setzer \& Saldívar del Ángel, 2012)

\subsection{1.-Tipos de análisis.}

Mencionando a Amaral, 2016, expone que existen dos tipos de análisis financiero:

Interno
-Análisis para fines
administrativos, el
encargado de hacer este
análisis es el
administrador financiero
que esta en constante
contacto con la empresa
para recabar los valores
contables e informacion
del negocio

Externo
-El analizador no tiene una
relación directa con la
organización, la única
información que conoce es
la proporcionada por la
empresa y la cual es
considerada pertinente

\subsection{2.- Métodos de análisis.}

Según el autor Perdomo 2003, considera un clasificación que engloba cinco métodos que se pueden aplicar en relación a lo que se desea analizar.

\begin{tabular}{|c|c|}
\hline \multirow[t]{5}{*}{$\begin{array}{l}\text { Vertical o } \\
\text { Estático }\end{array}$} & $\begin{array}{l}\text { Método para analizar un estado en una } \\
\text { fecha marcada o a un período en especifico }\end{array}$ \\
\hline & Procedimiento de porcientos integrales. \\
\hline & Procedimiento de razones simples. \\
\hline & Procedimiento de balance doble. \\
\hline & Procedimiento de razones estándar \\
\hline $\begin{array}{l}\text { Horizontal } \\
\text { o Dinámico }\end{array}$ & $\begin{array}{l}\text { Compara dos estados financieros de una } \\
\text { empresa a dos fechas o períodos } \\
\text { diferentes. }\end{array}$ \\
\hline
\end{tabular}

Procedimiento de aumentos y disminuciones

\begin{tabular}{|c|c|}
\hline \multirow[t]{4}{*}{ Histórico } & $\begin{array}{l}\text { Examina una sucesión de estados } \\
\text { financieros de la empresa en períodos } \\
\text { distintos }\end{array}$ \\
\hline & Serie de cifras o valores \\
\hline & Serie de variaciones \\
\hline & Serie de índices \\
\hline \multirow[t]{4}{*}{$\begin{array}{l}\text { Proyectado } \\
\text { o estimado }\end{array}$} & $\begin{array}{l}\text { Plantea diversos escenarios futuros de los } \\
\text { estados financieros proyectandolos a } \\
\text { fechas futuras. }\end{array}$ \\
\hline & Procedimiento del control presupuestal. \\
\hline & Procedimiento del punto de equilibrio \\
\hline & Procedimiento de control financiero Dupont \\
\hline \multirow[t]{3}{*}{ Bursátil } & $\begin{array}{l}\text { Se realiza para aquellos estados de } \\
\text { empresas que tienen acciones en la bolsa } \\
\text { de valores. }\end{array}$ \\
\hline & Análisis fundamentales. \\
\hline & Análisis Técnico \\
\hline
\end{tabular}

7.3.- Toma de decisiones. 
La importancia de la toma de decisiones permite a los generantes como a la organización en general, una mejor perspectiva de la problemática que se desarrolla y posibles alternativas de solución.

Es el proceso en el cual se identifican cuales son los problemas de una organización asi como sus áreas de oportunidad, proponiendo una serie de alternativas para la solución de estas. (Flore, 2015)

A continuación, se describen en que consiste cada una de las etapas de este proceso Robles, 2012.

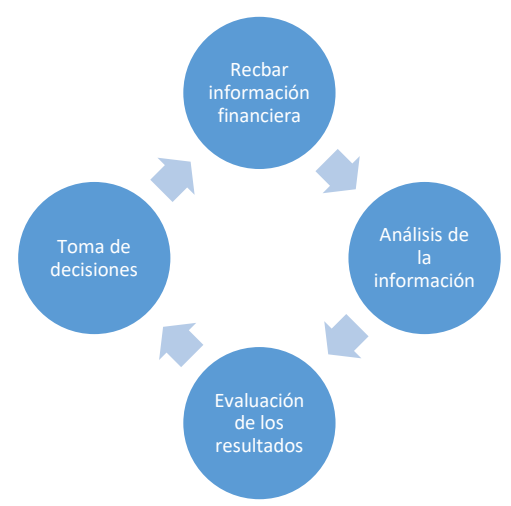

Mencionado al autor Padilla, (2016, pág. 335): Existen situaciones en las cuales es necesario la información financiera para determinar la elección mayor rentable para la organización, la metodología que se debería aplicar.

Se presentará la información de la situación que tienen la empresa, y la descripción de la situación a la que desea cambiar (utilizando ejemplos cuantitativos).

Se realizará el análisis marginal de las opciones y se seleccionará la mejor.

Se complementará el análisis con la información cualitativa, que deberá tomarse en consideración en cada circunstancia.

\section{8.- Equipo experimental}

Mediante la recaudación de información financiera de la empresa se empata la situación de la sociedad y la dimensión del problema, estudiar la información y usar instrumentos financieros para conocer en qué aspectos la empresa tiene problemas, evaluar los resultados que se obtuvieron para formular alternativas que mejoren las beneficios de la compañía, y la toma de decisiones que consisten en estrategias que permitan el mejoramiento de la organización.

\section{9.- Método}

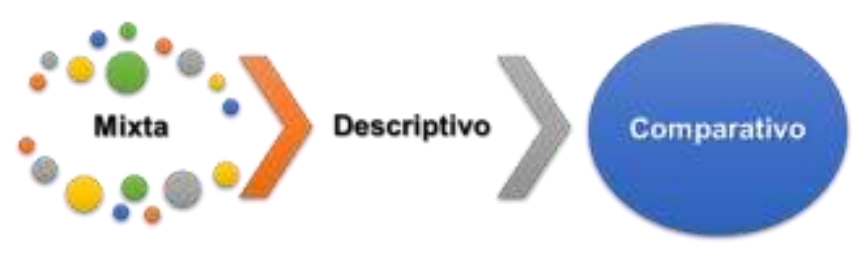

Es una metodología mixta porque representa el más alto grado de integración o combinación entre los enfoques cualitativo y cuantitativo.

Ambos se entremezclan o combinan en todo el proceso de investigación, o al menos, en la mayoría de sus etapas. Se necesita de un manejo completo de los dos enfoques y una mentalidad abierta. Agrega complejidad al diseño de estudio; pero contempla todas las ventajas de cada uno e los enfoques.

\subsection{Método de la investigación.}

Método descriptivo: permite la observación de un fenómeno en determinado tiempo y lugar, lo que permite conocer mejor la condicion de la compañía. (Arias Toma, 2014)

Este método será utilizado en el presente trabajo de investigación para describir los reportes económicos y financieros extraídos de los estados de resultados y estado de situación financiera, de periodos anteriores para analizar los datos económicos financieros y al final mostrar resultados.

Método comparativo: compara dos fenómenos semejantes, obteniendo resultados que seran de utilidad para su estudio. (Bernal, 2010)

Este método será utilizado para cotejar los rubros que componen el balance general y el estado de resultados.

\section{2.- Población y Muestra.}

La población del presente trabajo de investigación está dada por la empresa de transporte, que servirá como unidad de análisis. La muestra del presente trabajo de investigación será la misma que la población, tomando como base los estados financieros. 
La muestra no será necesario obtenerla debido a que el área de estudio es contabilidad y se podrá aplicar el instrumento a todos los elementos del área.

\section{0.- Resultados}

\section{1.- Razones financieras.}

\section{Capital de Trabajo}

\begin{tabular}{cccc}
\hline Año & Importe & Ratio \\
\hline \hline 2017 & $\$ 258,000,000-\$ 111,430,000$ & $\$$ & $146,570,000$ \\
& & & \\
2018 & $\$ 266,710,000-\$ 92,624,849$ & $\$$ & $174,085,151$ \\
\hline \hline
\end{tabular}

Interpretación: Podemos apreciar que su capital de trabajo incremento respecto al año 2017 en un 18.77\%, por lo que este índice muestra la suma con la cuenta la empresa para realizar sus procedimientos normales con $\$ 146^{\prime} 570,000.31$ en 2017 con incremento a $\$ 174^{\prime} 085,151.28$ para 2018.

\section{Liquidez}

\begin{tabular}{ccc}
\hline Año & Importe & Ratio \\
\hline \hline 2017 & $\frac{\$ 258,000,000}{\$ 111,430,000}$ & 2.3154 \\
& $\frac{\$ 266,710,000}{\$ 92,624,849}$ & \\
2018 & & 2.8795 \\
\hline \hline
\end{tabular}

Interpretación: Este indicador financiero también se incrementó respecto al año anterior, y esto le facilita la adquisición de materia prima, o una posible expansión de la empresa.

\section{Prueba de Ácido}

\begin{tabular}{ccc}
\hline Año & Importe & Ratio \\
\hline \hline \multirow{2}{*}{2017} & $\frac{\$ 258,000,000-\$ 2,110,000}{\$ 111,430,000}$ & 2.2974 \\
& $\frac{\$ 266,710,000-\$ 2,000,000}{2018}$ & \\
\hline \hline
\end{tabular}

Interpretación: Incremento de acuerdo con el año anterior, en el año 2017 por cada peso de deuda la empresa tenía \$2.30 para cubrirlo, y para 2018 aumento $\$ 0.56$, por lo cual se deduce que la empresa ha llegado a acuerdos favorables con sus proveedores, acreedores, etc.

\section{Cuentas por Cobrar}

\begin{tabular}{ccc}
\hline Año & Importe & Ratio \\
\hline \hline \multirow{2}{*}{2017} & $\frac{\$ 1,061,541,359}{\$ 105,000,000}$ & 36.1032 \\
& $\frac{\$ 1,153,387,552}{\$ 115,000,000}$ & \\
2018 & & 36.3928 \\
\hline \hline
\end{tabular}

Interpretación: Esta razón sigue manteniendo un promedio de 36 días de cobro para la empresa, por lo cual se deduce que la empresa no llegó a un acuerdo con sus clientes para acelerar la entrada de dinero.

Cuentas por Pagar

\begin{tabular}{ccc}
\hline Año & Importe & Ratio \\
\hline \hline \multirow{2}{*}{2017} & $\frac{\$ 1,036,051,306}{\$ 97,000,000}$ & 34.173 \\
& $\frac{\$ 1,119,319,067}{\$ 72,000,000}$ & \\
2018 & & 23.479 \\
\hline \hline
\end{tabular}

Interpretación: Analizando las razones de cuentas por cobrar y cuentas por pagar, apreciamos que en el año 2017 la empresa mantenía una misma longitud que su ciclo de cobro, pero para el año 2018 su periodo de pago es más anticipado de lo que cobra.

\section{Rotación de Inventarios}

\begin{tabular}{ccc}
\hline Año & Importe & Ratio \\
\hline \hline 2017 & $\frac{\$ 731,100,463.7}{\$ 2,000,000}$ & \\
& $\frac{\$ 807,036,147.6}{\$ 2,110,000}$ & \\
2018 & & 0.998 \\
\hline \hline
\end{tabular}

Interpretación: Esta razón mantuvo el mismo promedio los dos años, lo ideal sería lograr que los inventarios se mantengan en lo mínimo posible, donde en el almacén solo se tenga lo suficiente para cubrir los pedidos de los clientes y de esa forma no tener recursos representados en inventarios que no rotan o que lo hacen muy lentamente 
Endeudamiento

\begin{tabular}{ccc}
\hline Año & Importe & Ratio \\
\hline \hline 2017 & $\frac{\$ 131,430,000}{\$ 521,430,000}$ & 0.252 \\
& $\frac{\$ 117,624,849}{\$ 533,760,000}$ & \\
2018 & 0.220 \\
\hline \hline
\end{tabular}

Interpretación: En el año 2017 su endeudamiento era de $25.21 \%$ y para 2018 disminuyo a $22.04 \%$ lo cual significa que la empresa no tiene deudas excesivas, y que tiene capacidad para endeudarse si fuera necesario sin peligrar su nivel de solvencia y liquidez.

\section{Margen de utilidad}

\begin{tabular}{ccc}
\hline Año & Importe & Ratio \\
\hline \hline \multirow{2}{*}{2017} & $\frac{\$ 59,964,103.67}{\$ 1,061,541,359}$ & 0.056 \\
& $\frac{\$ 26,135,151.04}{\$ 1,153,387,552}$ & \\
2018 & 0.023 \\
\hline \hline
\end{tabular}

Interpretación: Representa el costo de oportunidad de los capitales que se mantienen en la empresa, y cuanto mayor sea su resultado, mejor será para la misma. Pero el resultado que arroja este índice muestra que su patrimonio sólo genera 0.0564 centavos por cada peso invertido en recursos propios en el 2017, reduciendo de manera desfavorable para el siguiente año 0.0227 centavos por cada peso invertido.

\section{ROI}

\begin{tabular}{ccc}
\hline Año & Importe & Ratio \\
\hline \hline \multirow{2}{*}{2017} & $(0.05648777)(2.035827165)$ & 0.115 \\
& & \\
2018 & $(0.02265947)(2.160872962)$ & 0.049 \\
\hline \hline
\end{tabular}

Interpretación: Es la capacidad básica de la entidad para generar utilidades por cada peso de Activo Total invertido, lo cual denota un nivel aceptable con $11.5 \%$ de eficacia de la gestión para el 2017, pero para 2018 tiene un declive cayendo al $4.89 \%$ sobre el $\mathrm{ROI}$.
ROA

\begin{tabular}{ccc}
\hline Año & Importe & Ratio \\
\hline \hline \multirow{2}{*}{2017} & $\$ 59,964,103.67$ & \\
& $\$ 1,061,541,359$ & 0.056 \\
2018 & $\$ 26135151.04$ & \\
\hline \hline
\end{tabular}

Interpretación: El resultado es el beneficio generado por el activo de la empresa, por cada unidad monetaria invertida en los activos la empresa obtiene 11.50 de unidades monetarias de utilidades netas para 2017, la cual se ve afectada para 2018 la cual disminuye al 4.90 de unidades monetarias de utilidades netas.

\section{2.- Modelo DuPont.}

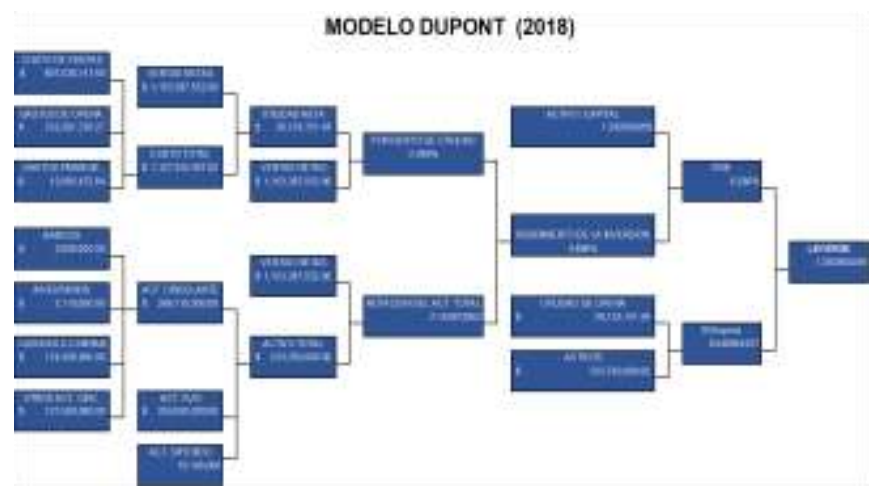

\section{3.- Propuestas de lineamientos económicos y financieros.}

Los ratios obtenidos en la rotación de cuentas por cobrar indican que se debe implementar una política para ejercer presión al departamento de crédito y cobranza, en solicitar anticipos a los clientes.

Se recomienda implementar el modelo Dupont, con el cual se consigue información de la situación financiera, indica su cometido, analiza rubros de las cuentas que compone la estructura de los estados financieros, establece indicadores financieros básicos y analiza e interpreta los resultados, evidenciando la posición financiera, facilitando la toma de decisiones.

Se sugiere a la agrupación de transporte la evaluación de su información financiera por los responsables del área, que le permitan tomar decisiones futuras para mantener la permanencia económica de la organización en el mercado. 


\section{1.- Discusión}

El análisis financiero constituye uno de los métodos más habituales que usan las organizaciones para tomar decisiones.

Los estados financieros muestran cuál es la realidad económica de la empresa, así como cuáles son los indicadores financieros en se debe buscar una mejora.

El balance general, estado de resultados, modelo DuPont, son parámetros que sirven para evaluar la condición de la empresa, y es así como se identificara que variables afectan el desempeño de la organización. Trabajos de investigación anteriores mencionan que "El análisis financiero se debe realizar con el uso de las ratios financieros para su posterior y correcta evaluación y se debe tener en cuenta estos índices de tal manera que sean sustento para mejorar las deficiencias en la Empresa como es la implementación de una adecuada política financiera para que no afecte a la rentabilidad".

Frecuentemente se hace un incorrecto análisis financiero porque los directivos desconocen la existencia de herramientas financieras, o solo porque toman decisiones por instinto, sin indicadores que lo respalde.

\section{2.- Conclusiones}

La investigación realizada contribuye de manera importante para identificar como se debe llevar a cabo un correcto análisis financiero para tomar decisiones

De acuerdo con los resultados obtenidos en base al análisis financiero, se concluye que un correcto estudio de los indicadores financieras en la empresa de transporte, ayuda a la alta dirección al momento de tomar decisiones, al aplicar las razones financieras brinda la oportunidad de detectar cuales son las necesidades de una empresa ofreciendo diferentes escenarios e influyendo en el funcionamiento de la organización y asi cumplir con los objetivos de la organización.

\section{Referencias}

Amaral, A. W. (2016). Herramientas financieras: un instrumento para la toma de decisiones en una empresa del sector salud. Mexico df: instituto politécnico nacional.

Armenteros, D. C. (2018). El análisis de los estados financieros: papel en la toma de decisiones gerenciales. Artículo, Universidad de Ciego de Ávila "Maximo Gómez Báez". Obtenido https://www.researchgate.net/publication/239950588

Bernal, C. A. (2010). Tipos de investigación. En C. A. Bernal, Metodología de la investigación (Tercera ed., pág. 320).
PEARSON. Obtenido de biblioteca.uccvirtual.edu.ni/index.php?Option=com_docman $\& \operatorname{task}=\mathrm{doc}$

Coyla Limachi, M. J. (2015). El análisis financiero para la toma de decisiones en la empresa Operadora Surperu, S.A, peirodos 2012 - 2013. Tesis, Universidad Nacional del Antiplano, Puno. Perú. Obtenido de http://repositorio.unap.edu.pe/bitstream/handle/U NAP/2018/Coyla_Lmachi_Mario_Jimmy.pdf?seq uence $=1 \&$ isAllowed $=\mathrm{y}$

Flores, N. (2015). Estrategías financieras aplicadas al proceso de sostenibilidad y crecimiento de las PYMES del sector comercial del municipio Santiago del Estado Aragua. Tesis de Maestria, Universidad de Carabobo, Facultad de Ciencias Económicas y Sociales. Maestría en Administración de Empresas. mención Finanzas, La Morita. Obtenido de http://mriuc.bc.uc.edu.ve/bitstream/handle/123456 789/2536/Maestr\%C3\%ADa\%20Nancy\%20Flores .pdf?Sequence $=1$

Hurtado Romero, L. J. (2014). La gerencia financiera en la toma de decisiones. Tesis, Universidad Militar Nueva Granada, Facultad de Ciencias económicas. Contaduría Pública, Bogotá. Obtenido de http://mriuc.bc.uc.edu.ve/bitstream/handle/123456 789/2536/Maestr\%C3\%ADa\%20Nancy\%20Flores .pdf?Sequence $=1$

Ochoa Setzer, G. A., \& Saldívar del Ángel, R. (2012). Ánalisis financiero. En G. A. Ochoa Setzer, \& R. Saldívar del Ángel, Administración Financiera. Correlacionada con las NIF (pág. 736). Distrito Federal, México: McGraw Hill.

Padilla, D. N. (2016). El papel de la contabilidad administrativa en la toma de decisiones. En D. N. Padilla, Contabilidad administrativa (Octava ed., pág. 626). México, D.F.: MCGraw Hill. Obtenido de http://www.resistenciacontable.org.py/index.php/d escargas/libros?download=53: contabilidadadministrativa

Robles, R. C. (2012). Fundamentos de Aaministración Financiera. (Primera, Ed.) Estado de México, DF, México: Red Tercer Milenio. Obtenido de https://es.scribd.com/doc/147732256/Fundamento s-de-Administracion-Financiera 